\title{
ProPSMA: A Callout to the Nuclear Medicine Community to Change Practices with Prospective, High-Quality Data
}

\author{
Michael S. Hofman \\ Molecular Imaging and Therapeutic Nuclear Medicine, Peter MacCallum Cancer Centre, Melbourne, Australia; and Sir Peter \\ MacCallum Department of Oncology, University of Melbourne, Melbourne, Australia
}

W pertate-specific membrane antigen (PSMA) PET/CT examination in mid-2014, and it was immediately obvious that this new imaging modality was vastly superior to the existing practice of CT and bone scanning or of choline PET/CT. This first patient was scheduled to undergo a prostatectomy with curative intent, but the PSMA PET/CT additionally demonstrated pelvic nodal and distant osseous metastatic disease (Fig. 1). His management was changed, and he was enrolled in a clinical trial of enzalutamide. When I met this man, who was in his late 70s, briefly after a follow-up scan 1 y later, he remained symptom-free and was pleased to have forgone the risks of surgery when it had no chance of cure.

The striking images we are now able to produce in nuclear medicine and the precision medicine we practice are not, however, enough to convince governments or insurers to either approve or fund such new technologies. Unbiased comparative effectiveness data comparing PSMA PET/CT with existing technology are a necessary prerequisite. We need to be able to provide precise data on accuracy, management impact, reproducibility, and safety compared with currently used technologies and show health economic benefits.

In late 2015, through the Australasian Radiopharmaceutical Trials network, we expressed interest in a grant opportunity from the Prostate Cancer Foundation of Australia funded through Movember. Back then, the world had barely heard of PSMA PET/CT. In close collaboration with our urology experts, radiation oncology experts, clinical trials experts, and consumer advocates, we put together a successful grant proposal culminating in the proPSMA study (1). We established a network of 10 sites around Australia with the capability to perform on-site radiopharmaceutical production and PET acquisitions according to standardized methods (2). The study recruited well, and we expanded it to 300 patients to ensure it was adequately powered. Referrers were engaged, and recruitment was completed more than 6 mo ahead of schedule. A huge amount of data was collected, including an imaging biobank that will form the basis of further research.

The ProPSMA clinical trial results, published in The Lancet (3), show that ${ }^{68} \mathrm{Ga}$-PSMA-11 PET/CT offers greater accuracy than conventional imaging, with an accuracy of $92 \%$ for detecting pelvic nodal or distant metastases, compared with $65 \%$ for standard

Received Mar. 21, 2020; revision accepted Mar. 23, 2020.

For correspondence or reprints contact: Michael S. Hofman, Peter MacCallum Cancer Centre, Locked Bag 1, A'Becket St., Melbourne, Victoria, Australia 8006.

E-mail: michael.hofman@petermac.org

Published online Apr. 3, 2020.

COPYRIGHT (C) 2020 by the Society of Nuclear Medicine and Molecular Imaging. DOI: 10.2967/jnumed.120.245647 imaging with the combination of CT and bone SPECT/CT (Fig. 2). Moreover, PSMA PET/CT led to management changes-defined by a change in treatment modality or a change in delivery-in $28 \%$ of men, compared with $15 \%$ for conventional imaging. Other outcomes showed fewer equivocal (uncertain) findings (7\% vs. $23 \%)$, half the radiation dose ( 8.4 vs. $19.2 \mathrm{mSv}$ ), and high reporter agreement for PSMA PET/CT. When PSMA PET/CT was performed after conventional imaging as a second-line imaging test, it had a similarly high rate of change in patient management. Conversely, just $5 \%$ of patients had their treatment plans modified with second-line CT or bone scans, and only $2 \%$ had an accurate change in their stage with CT or bone scans.

By now, hundreds of thousands of PSMA PET/CT examinations have been performed globally. Doctors with experience in adopting this new technology generally have no doubt that it is a high-impact modality and superior to conventional imaging. More than 1,500 articles on it have been published, mostly retrospective analyses without comparison to a reference standard. By comparison, the proPSMA study has imaged a small number of men-only 300but has resulted in a rich dataset that has generated definitive data. This study will hopefully provide the necessary data to achieve reimbursement for PSMA PET/CT, enabling widespread availability for men globally.

Precision medicine has often been at variance with evidencebased medicine. In the former, individual results inform patient

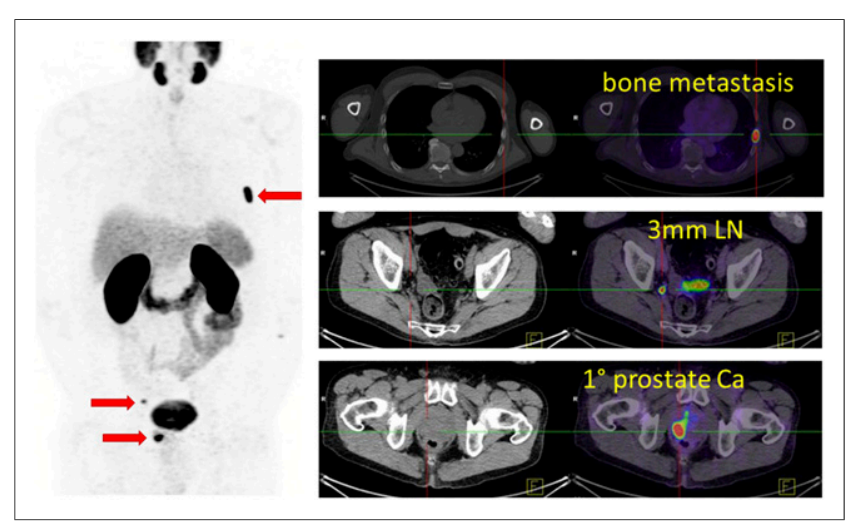

FIGURE 1. Man with newly diagnosed Gleason grade group 4 prostate cancer, with no evidence of metastatic disease on CT, bone scanning, or MRI of pelvis (not shown). PSMA PET/CT demonstrated $<5-\mathrm{mm}$ pelvic nodal metastases, the primary tumor, and bone metastasis (red arrows). Patient's management was directed away from curative-intent prostatectomy. 


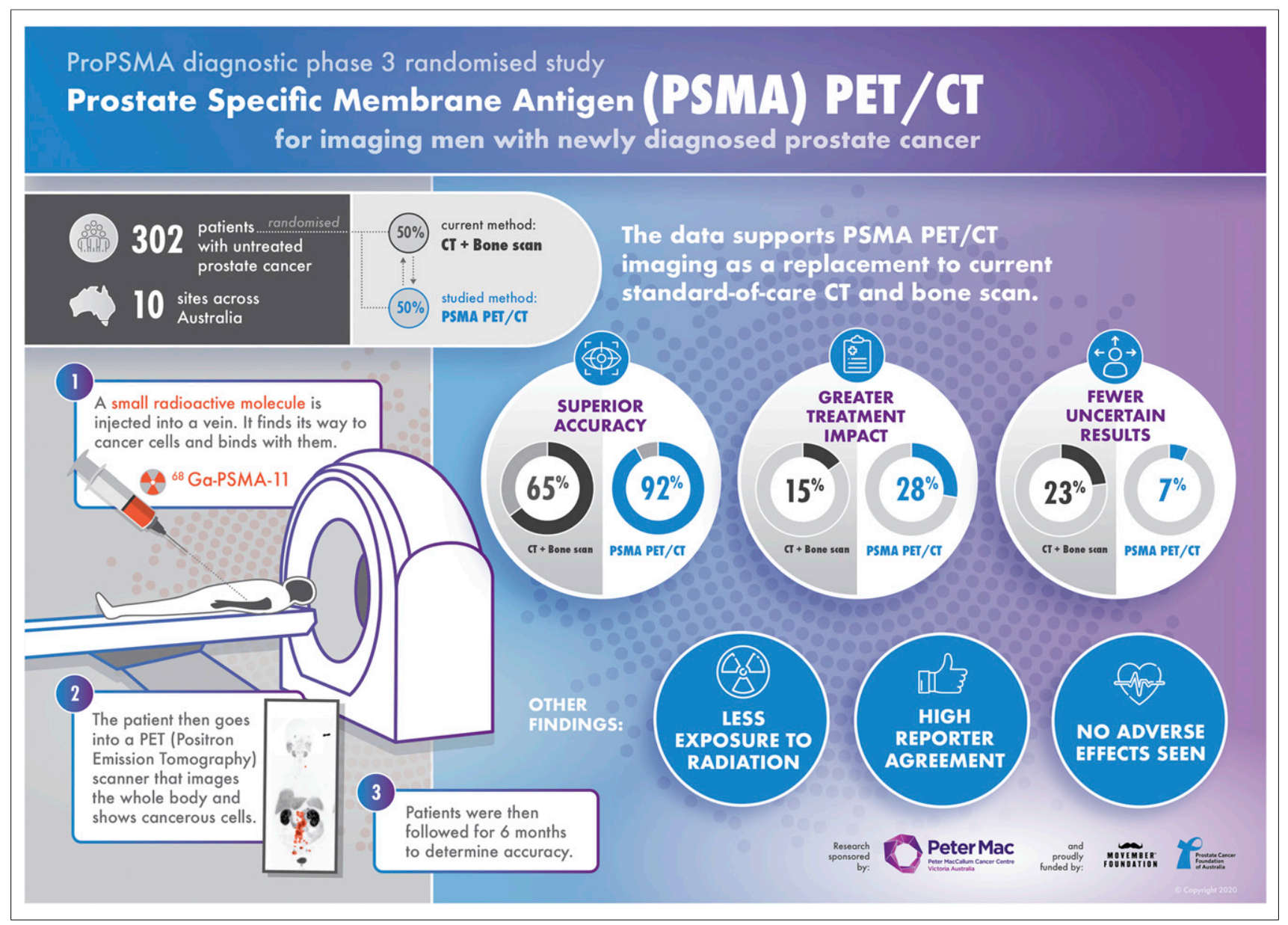

FIGURE 2. Infographic summarizing proPSMA clinical trial results.

management, whereas in the latter, results are informed by randomized controlled trials and metaanalyses. ProPSMA tries to bridge the gap between these 2 worlds. As nuclear medicine specialists, we need to generate more unbiased, prospective, multicenter data. Establishing collaborative networks, working together, and upskilling the next generation of specialists in clinical trial methodology are key to achieving this goal. As new radiotracers emerge, there is a pressing need to develop and enroll patients in well-designed clinical trials. These are our best chance to properly evaluate the impact of our tests and enable widespread access by patients.

\section{DISCLOSURE}

The ProPSMA study was supported by a clinical trials award from the Prostate Cancer Foundation of Australia (PCFA), funded through Movember and sponsored by the Peter MacCallum Cancer Centre. Michael Hofman was additionally funded during the writing of this editorial by the Peter MacCallum Foundation (Clinical Fellowship Award), Prostate Cancer Foundation (PCF), Australian Government (Medical Research Future Fund), Victorian Cancer Agency, and the U.S. Department of Defense. Unrelated to this work he has received personal fees and nonfinancial support from Jannsen, Ipsen, and Sanofi Genzyme. No other potential conflict of interest relevant to this article was reported.

\section{ACKNOWLEDGMENTS}

Michael Hofman thanks all the ProPSMA study investigators, clinical trial coordinators, participants and their carers, for all their dedicated efforts in the conduct of the ProPSMA Study. He also thanks the Australian and New Zealand Urogenital and Prostate Cancer Trials Group (ANZUP) and The Australasian Radiopharmaceutical Trials network (ARTnet) for supporting the trial.

\section{REFERENCES}

1. Hofman MS, Murphy DG, Williams SG, et al. A prospective randomized multicentre study of the impact of gallium-68 prostate-specific membrane antigen (PSMA) PET/ CT imaging for staging high-risk prostate cancer prior to curative-intent surgery or radiotherapy (proPSMA study): clinical trial protocol. BJU Int. 2018;122:783-793.

2. Bailey DL, Hofman MS, Forwood NJ, et al. Accuracy of dose calibrators for ${ }^{68} \mathrm{Ga}$ PET imaging: unexpected findings in a multicenter clinical pretrial assessment. $J$ Nucl Med. 2018;59:636-638.

3. Hofman MS, Lawrentschuk N, Francis RJ, et al. Prostate-specific membrane antigen PET-CT in patients with high-risk prostate cancer before curative-intent surgery or radiotherapy (proPSMA): a prospective, randomised, multi-centre study. Lancet. March 20, 2020 [Epub ahead of print]. 